\title{
OTSA: Optimized Time Synchronization Approach for Delay-based Energy Efficient Routing in WSN
}

\author{
K. Nagarathna \\ Research Scholar \\ Visvesvaraya Technological University \\ Belagavi, Karnataka
}

\author{
Jayashree D Mallapur \\ Dept. of Electronics \& Communication Engg \\ Basaveshwar Engineering College \\ Bagalkot, Karnataka, Iwendia
}

\begin{abstract}
Time Synchronization is one of the problems and still ignored problem in area of wireless sensor network (WSN). After reviewing the existing literatures, it is found that there are few studies that combinely address the problem of energy conservation, clustering, routing along with minimizing the errors due to time synchronization in sensor network. Therefore, this manuscript presents a delay-based routing which considers the propagation delay to formulate a mechanism for delay compensation in large scale wireless sensor network. The prime goal of this technique is to jointly address energy problems, time synchronization, and routing in wireless sensor network. The outcome of the proposed study was found to posse's minimized communication overhead, minimized synchronization errors, lower energy consumption, and reduced processing time when compared with the existing standards of time synchronization techniques.
\end{abstract}

Keywords-Wireless Sensor Network; Routing; Time Synchronization; Optimization; Hardware Clock

\section{INTRODUCTION}

Wireless sensor network is increasing finding its utility in unmanned monitoring system over hazardous geographical locations [1]. Normally sensors are positioned in those area where is inaccessible for humans. Various applications of sensors network includes habitat monitoring, healthcare monitoring system, industrial monitoring system etc. In all the application, time is one of the important factors to guarantee the communication success. Two decades ago, the applications of the sensor network were more focused on just delivering the remote data. However, with the changing times, the information has become more time bound in terms of delivery. Another change in modern sensor network is the data quality in sensor network, which is only possible when an effective time stamp is given. Hence, time plays an extremely critical role in providing data quality as well as Quality-of-Service. However, there is one impediment in this regards, which is called as time synchronization [2] [3]. It is essentially use to map as well as synchronize all the hardware clocks residing in the sensors. Time synchronization plays an important role in wireless sensor. The hardware clock in the senor node has an inbuilt oscillator that is responsible for generating pulse. The problems start shooting when the sensor present in the environment start exhibiting different local time with higher difference in their values. In such cases, the two sensors sense the same event at same time but time stamps it with different value. Such problem gives rise to all sort of data quality issues that poses a potential threat to both communication performance as well as security [4][5]. The problem of time synchronization is often studied with respect to some standard terms e.g. clock offset, clock drift, and clock synchronization. Clock offset can be stated as just the different of local clocks with respect to its local time. Clock drift can be stated as frequency difference of the logical clocks. The originations of the time difference occur when sensors are turned on very frequently at arbitrary intervals.

In order to study time synchronization, various research work have been already carried out in the last decade. In such research work, various performance parameters selected by the researchers are accuracy, precision, energy consumption, fault tolerance, and memory utilization. However, there are various constraints which the prior research work have been highlighting pertaining to the time synchronization viz. i) different forms of precision needs, ii) scalability to large scale computing, iii) minimal mobility, iv) non-regularization of higher value of throughput or delivery ratio, v) insignificant transmission delay among the adjacent sensors. An effective algorithm for time synchronization should permit the sensor to be in synchronized state around the clock. The prime idea is also restore maximum amount of energy consumption which finally leads to maximum level of energy drainage. There is various applications where time synchronization plays a critical role in delivering quality services e.g. healthcare. A minor form of error in received message will cost the life of patient. Hence, still the research community is emphasizing on this issues as sensors are increasingly used in healthcare sector. Hardware availability has improved keeping back the appropriate software / protocol availability.

Therefore, the present manuscript presents a novel optimized algorithm for time synchronization with equal emphasis on energy efficiency as well as delay-based routing in large scale WSN. The outcome of this paper is an energy efficient routing protocol that reduces synchronization error in WSN with benchmarked outcomes to prove its effectiveness. Section II discusses about the prior research work carried out in the area of time synchronization followed by discussion of problem identification in Section III. Proposed system is discussed in Section IV followed by research methodology adopted for the designing it in Section V. Algorithm implementation is discussed in Section VI followed by discussion of comparative analysis result accomplished from the study in Section VII. Finally, Section VIII makes some concluding remarks. 


\section{RELATED WORK}

This section discusses about the existing studies being carried out in the problem area of time synchronization pertaining to wireless sensor network. In prior study [6] have discussed about various techniques adopted and introduced in past for reducing the synchronization error in sensor network.

Xiong et al. [7] have developed a technique that assists in performing localization in sensor network. The presented technique has implemented standard Gaussian noise model for estimating the time difference from the detection with the targeted object and the sensors. Although, the author have not directly used time synchronization concept, but the technique is meant for eliminating the difference of time among the communicating nodes in case of localization application in sensors. Xing et al. [8] have developed a novel synchronization technique using linear approach. Linear predictive-based frameworks have been introduced by the author where the prediction estimation is carried out using statistical parameters. Using synchronization model of master and slave, the proposed system minimizes the synchronization errors. Ting et al. [9] have developed a state-spaced framework in order to establish a persistent relationship among the communicating nodes using control strategies. The study has even used an iterative Kalman filter. Kim and Yoo [10] have presented a scheme of time synchronization in sensor network considering the challenges of reducing errors occurring undersea bed. The controlling strategy of synchronization is developed by using mobility factor and node usage. Kalman filter is still found to be used in this mechanism for minimizing the localization errors. The outcome of the study is analyzed with respect to position in particular direction with respect to elapsed time, error in skew value, localization error, etc.

Jin et al. [11] have used voltage factor in minimizing errors causing due to time synchronization. The technique initially identifies the extent of skewness in clock and performs updating operating on local clocks only based on extent of residual voltage. The technique that fine tunes the resynchronization arbitrarily depending on the rate of errors occurring in controlling and balancing the synchronization errors. Dengchang et al. [12] have applied the logic of maximum consensus in order to compensate clock drift as well as mean consensus for compensating offset of clock. The author had presented the work in the form of mathematical modelling and graph theory to find reduced synchronization error as an outcome. Wang et al. [13] have presented a mechanism that can perform synchronization of time along with supportability of multi-hop communication in sensor network. The author have perform rectification of time synchronization problems on two hops for minimizing an adverse effect of overhead and furnishing seamless synchronization to all nodes in dynamic networks. Ardakani et al. [14] have presented a unique algorithm that can perform ondemand time synchronization in sensor network. It is a kind of reactive protocol that performs minimization of time synchronization errors along with exponential minimization of network overheads.

A standard study towards computing time synchronization is carried out in sensor network considering the distributed fashion by Stankovic et al. [15]. The authors have presented a stochastic based technique in order to compensate the evaluated clock drift and offsets. The uniqueness of the study is that it attempts to minimize synchronization errors in presence of noise using mathematical modelling approach. Youn [16] have also studied about the clock synchronization problems in sensor network. Some of the interesting findings include usage of MAC layer for performing time stamping. The authors have also advocated about the usage of the linear approach for truncating the failures of the local clocks in sensor network thereby allowing the system to perform effective time synchronization. Kerkez [17] have presented another unique study that emphasizes on the adaptive fashion of synchronizing the sensor nodes. The authors have used channel hopping scenario with enhancing the MAC layers. Applicability of this technique is found to be highly suitable for mobile agents in sensor network for minimizing errors. A similar form of study is carried out by Wang et al. [18] who have also used MAC layer time stamping mechanism using real-time sensors. The authors have used the technique over TinyOS where the outcomes were evaluated with respect to precision value in presence / absence of compensation techniques. Ikram et al. [19] have presented an idea where time synchronization is carried out in sensor network using radio resources where the study particularly focuses on reducing the errors occurring due to probabilistic sources. The study uses hardware-based approach to justify the claims of minimizing time synchronization. Gautam and Sharma [20] have presented a study that emphasizes on comparative analyses of various techniques used for time synchronization in sensor network.

Lenzen et al. [21] have presented a standard model of time synchronization called as PulseSync that is designed to rectifying the errors in large scale sensor network. Implemented in hardware platform in TinyOS, it is found to offer better accuracy with respect to existing flooding based approaches. Security was also incorporated with respect to synchronization issues by Sanchez et al. [22]. The study uses a predictive-based technique with exchange of synchronization beacons in body area network. Another standard model was presented by Tang et al. [23] that uses multiple channel MAC protocol for performing time synchronization in sensor network with emphasis on energy efficiency. The experiment was carried out over real-time Mica $\mathrm{Z}$ sensors with respect to packet delivery ratio, latency, and duty cycle. Albu et al. [24] have presented a study that jointly enhances the clock synchronization and energy efficiency in sensor networks. The study was performed in network simulator to accomplish the better accuracy.

Hence, it can be seen that there are various studies being carried out toward time synchronization in wireless sensor network, which essentially contributes to formulate novel idea for future enhancement. The next section discusses about the inherent limitations in the existing literatures, which will be addressed in present manuscript as proposed study.

\section{PROBLEM IDENTIFICATION}

This section discusses about the problems that are found to be likely unaddressed in existing techniques. The proposed study find that there are various techniques in past where 
Kalman filtering is deployed for ensuring better accuracy in time synchronization in sensor network. Fundamentally, Kalman filter is best suited for any state which is continuously changing. However, how this change will be required to be updated to all the nodes in a large scale sparsely populated sensors is still an unsolved question. Researchers have not found any substantial evidence that usage of Kalman filter can also affect simultaneous updates of continually changing values of local clock nor it assists in energy preservation owing to its iterative process of monitoring the changes in clock drifts.

One of the most essential parts of the time synchronization is its capability to discretize time between two nodes located at two different distances. In majority of the conventional studies, the synchronization is carried out by the reference node that is responsible for estimating the drift value as well as offset value of the reference clock as well as local clock. Unfortunately, in order to maintain the accuracy of the clock, it is essential that propagation delay should be considered. This phenomenon causes although better precision, but it overlooks various noncommunicating nodes in the sensor network. If the delay calculation is carried out for transferring the values of the local clock readings from one to another node that it would be able to perform compensation of the transmission delay. Unfortunately, the existing studies are more inclined towards compensation of drift and offset and less focus on compensating the propagation delay. It is also possible that there could be serious synchronization errors owing to the delay of message transmission from the neighbour nodes if they are located very far away from the reference node. A missing gap in this assumption is clusterhead and its charecteristics.

Another research gap found in this context is enhancing the network lifetime. In order to combinely address time synchronization problems and energy efficiency, it is required that retransmission interval as well as ratio of synchronized messages with respect to cumulative message be considered as a part of delay calculations with network updates. However, all these are independent problems which still were not addressed with one single algorithm in past or recent studies in sensor network. It has not come across any potential research work towards time synchronization that has focused on data aggregation problems, clustering problems, and time synchronization problems jointly. Considering of transmission delay posses various complexities in design principle of time synchronization in wireless sensor network. It is still an unsolved problem to consider delay factor in optimization of time synchronization technique in sensor network.

Hence, the problem statement of the proposed study could be stated as "It is a computationally challenging task to develop a novel optimized algorithm that can consider delay factor in data aggregation to ensure energy efficient time synchronization in sensor network." The next section discusses about the proposed study that targets to eliminate the explored problems pertaining to time synchronization.

\section{PROPOSED SYSTEM}

The prime aim of the proposed study is to formulate a novel optimization technique that can combinely address the problem of i) time synchronization, ii) enhancement of network lifetime, and iii) data aggregation for large scale wireless sensor network. It is Optimized Time Synchronization Algorithm or OTSA, where the optimization is a terms that is more associated with delay-based routing to enhance network lifetime and significantly reduce errors occurring in time synchronization. This work is a continuation of the prior techniques [25][26][27][28][29] for the similar reason. The schematic architecture of the proposed system is showcased in Fig.1.

\begin{tabular}{|c|c|c|c|c|}
\hline \multicolumn{5}{|c|}{ Sensor Parameters } \\
\hline \multicolumn{5}{|c|}{$\mathrm{E}^{2} \mathrm{TS}$ Framework } \\
\hline \multirow{3}{*}{$\begin{array}{l}\text { Algorithm } \\
\text { for } \\
\text { Estimating } \\
\text { Real-Clock } \\
\text { Time }\end{array}$} & $\begin{array}{l}\text { Principle } \\
\text { Frequency }\end{array}$ & $\begin{array}{r}\text { Offset o } \\
\text { Frequ }\end{array}$ & $\begin{array}{l}\text { Clock } \\
\text { ancy }\end{array}$ & $\begin{array}{c}\text { Drift of Clock } \\
\text { Frequency }\end{array}$ \\
\hline & \multicolumn{4}{|c|}{ Modeling Hardware Clock } \\
\hline & \multicolumn{2}{|c|}{ Clock Offset } & \multicolumn{2}{|c|}{ Clock Skew } \\
\hline \multirow{3}{*}{$\begin{array}{l}\text { Algorithm } \\
\text { for } \\
\text { Constructing } \\
\text { Delay-Based } \\
\text { Message }\end{array}$} & \multicolumn{2}{|c|}{$\begin{array}{c}\text { Message for Query/ } \\
\text { Selection of } \mathrm{CH}\end{array}$} & \multicolumn{2}{|c|}{$\begin{array}{c}\text { Message for Request } \\
\text { to Perform } \\
\text { Synchronization }\end{array}$} \\
\hline & \multicolumn{4}{|c|}{ Response Message for Synchronization } \\
\hline & \multicolumn{4}{|c|}{ Local Time Stamping Parameters } \\
\hline \multicolumn{5}{|c|}{ Algorithm for Delay-Based Routing } \\
\hline \multicolumn{5}{|c|}{ Algorithm for Delay Compensation } \\
\hline
\end{tabular}

Fig. 1. Schematic Architecture of OTSA

The contribution of the proposed OTSA is as follows:

- To develop a novel delay-based routing that considers transmission delay and formulates the estimation procedure of drift, skew, and offset.

- To develop a completely new type of control message that can extract information pertaining to time synchronization between clusterhead and member nodes.

- To develop a robust technique that can explore the network dynamics and can allow the node to formulate strategy for delay compensation along with enhancement of network lifetime.

- To perform a comparative analysis of the proposed system with the existing approaches of the time synchronization on multiple performance parameters.

The next section of the study will discuss about the research methodology being adopted for developing the proposed study. 


\section{RESEARCH METHODOLOGY}

The proposed study considers analytical research methodology to be incorporated in the clock model. While developing the proposed system that all the broadcasting beacons to be time stamped only at the MAC layer. However, bring their a new mechanism of considering the local time stamping mechanism, which the conventional studies on time synchronization are not found to posses. The local time stamping mechanism considers multiple states of the sensor to forward request or to receive the response. Such abundant time parameters $\left(\mathrm{T}_{\text {for, }} \mathrm{T}_{\text {rec, }}, \mathrm{T}_{\text {res, }}, \mathrm{T}_{\mathrm{CH} \_ \text {res }}\right)$ are considered for delay considerations. It's developed their proposed OTSA over prior $E^{2} T S$ [29] framework itself considering the enhancement of the network lifetime.

The proposed system initiates by modelling the hardware clock considering the computation of skew as well as drift value over network dynamics. In order to emphasize on the delay-based routing, it is formulated 3 different forms of message viz. query message, request message, and response message. In order to specifically infer the meaning of the control message, it uses positive and negative values in the messages. The control message for OTSA will consists of i) type of beacon, which is a message pertaining to query or selection of clusterhead, ii) source node ID, iii) destination node ID, iv) intermediate node ID, v) clusterhead identity, vi) timestamp at message forwarding process, v) timestamp at message receiving process, vi) timestamp at response forwarding process, vii) time at which the $\mathrm{CH}$ forwards the response. One novelty of OTSA design is its independence from graph theory as it has its more applicability on multiple forms of network topology. Any node in the network who wants to communicate will be require to broadcast the above mentioned control message. The other node (it can be destination or intermediate node) than responds according sharing the transmission delay information which will be required to be compensated at the end of the processing.

The delay based-routing works as follows - whenever a sensor has received a control message, it checks if the received control message is query type or selection of clusterhead type. In case, if the received message is of query type, than it forwards the response with clusterhead identity or else it check for of the identity of the clusterhead is register in their routing table or not. In case of unidentified identity of clusterhead, the new identity is added in the routing table as a process of updating or else, it check if the residual energy of the cluster head is more or less to perform this communication. However, if the inbound control message is a request message, it checks for identity of the node to clusterhead or member node. Even the system finds that inbound control message is neither a query / selection, nor request message, than it checks if the inbound message is response message. In case of positive identification of response message, the node estimates transmission delay and add the local time stamping data with the message to be prepared for response message. In case of presence of an intermediate hops, the algorithm forwards the response to the prior sender. In a nutshell, the prime task in this stage is to ensure that proper identification of the control message being made that ends with calculations of transmission delay and local time stamping parameters to be incorporated as a measure of delay compensation strategy. In the entire process, the proposed system implements a first order radio model just to calculate energy, but in order to enhance the network lifetime, the OTSA technique ensures that that messages to be sent by run-time verification of the delay. This phenomenon will result in instant message propagation resulting in better Quality-of-Services. This process also results in minimizing the probability of retransmission that significant restores the transmission energy.

The proposed OTSA technique also ensures proper selection of clusterhead, which is extremely different from any existing techniques of selection. It is to be noted that every node to start the minimization process of synchronization request depending on the skew. Interesting, the proposed system also ensures that an algorithm provides better information of the global time, which is required for updating the clock drifts. Hence, delay estimation significantly assists in overcoming the major deviations in the time synchronization. In order to save energy, threshold-based energy has been used, so that when the clusterhead reaches the threshold value of energy, it is simply treated as member nodes. In such cases algorithm makes prior selection of clusterhead based on delayoriented routing in large scale wireless sensor network. Table 1 highlights the notations being used in the proposed study.

TABLE I. NOTATION USED

\begin{tabular}{|l|l|}
\hline New & Meaning \\
\hline$\lambda_{\mathrm{t}}(\mathrm{t})$ & Frequency of clock \\
\hline $\mathrm{t}$ & Principle time \\
\hline$\lambda_{\mathrm{o}}$ & Principle frequency \\
\hline$\Delta \lambda$ & Offset of clock frequency \\
\hline$\delta_{\lambda}$ & Drift of clock frequency \\
\hline$\varepsilon_{\lambda}(\mathrm{t})$ & Random error of clock \\
\hline $\mathrm{h}_{\mathrm{i}}(\mathrm{t})$ & Hardware clock \\
\hline $\mathrm{O}_{\mathrm{i}}$ & Clock Offset \\
\hline $\mathrm{S}_{\mathrm{i}}$ & Clock Skew \\
\hline $\mathrm{m}_{1}$ & Message for Query/Selection of CH \\
\hline $\mathrm{m}_{2}$ & Message for Request to Perform Synchronization \\
\hline $\mathrm{m}_{3}$ & Response Message for Synchronization \\
\hline$l t$ & Local time stamping parameters \\
\hline$d$ & Delay \\
\hline$E_{\text {res }}$ & Residual energy \\
\hline$T h$ & Cut-off residual energy \\
\hline $\mathrm{T}_{\mathrm{for}}$ & timestamp at message forwarding process \\
\hline $\mathrm{T}_{\text {rec }}$ & timestamp at message receiving process \\
\hline $\mathrm{T}_{\text {res }}$ & timestamp at response forwarding process \\
\hline $\mathrm{T}_{\mathrm{CH} \_ \text {res }}$ & Time at which the CH forwards the Response \\
\hline
\end{tabular}

\section{ALGORITHM IMPLEMENTATION}

This section discusses about the algorithm that has been implemented for developing the proposed study. The design of the proposed system is carried out considering 4 different algorithms for the purpose of rectifying errors in synchronization and performs delay-based routing with enhancement of network lifetime in large scale wireless sensor network.

\section{1) Algorithm for estimating real-clock time}

Although the proposed study is carried out using simulation approach, but still attempt to perform estimation of real clock time, which is basically the hardware clock time. The algorithm takes input of $\lambda_{\mathrm{o}}$ (principle frequency), $\Delta \lambda$ (offset of clock frequency), and $\delta_{\lambda}$ (drift of clock frequency). A temporary 
variable $\mathrm{x}$ is used in order to get the added value of $\Delta \lambda$ (offset of clock frequency), and $\delta_{\lambda}$ (drift of clock frequency), which is the prime deviation in clock value from the global values of other sensors present in the simulation area (line-2). The novelty of this algorithm is it considers modelling of hardware clock considering both drift and offset. Another novelty of this algorithm is consideration of the error. As owing to various forms of hardware-related issues, there could be possibility of slight errors in the clock values. This may be also due to related interfering operations in same sensors. Hence, It consider the error $\varepsilon_{\lambda}(\mathrm{t})$ to be a random type and add it with the adopted frequency of a clock. Therefore, line- 4 is valid only for the cases of local clocks that don't have much dependency on any significant issues related to hardware. Line-5 shows the test-observation time considered for the hardware clock $h_{i}(t)$ with the new variable $y$ representing ratio of offset of clock frequency to principle frequency. Finally, considering zero value of error, It normalize to look like line- 6 consisting of clock offset $\left(\mathrm{O}_{\mathrm{i}}\right)$ and clock skew $\left(\mathrm{S}_{\mathrm{i}}\right)$. It is to be noted that value of clock skew parameter $S_{i}$ lies between $(1-r)$ and $(1+r)$, where $r$ is optimal rate of drift of hardware clock.

\section{Algorithm for framing clock component}

Input: $\lambda_{\mathrm{o}}, \Delta \lambda, \delta_{\lambda}$

Output: hardware clock time

\section{Start}

1. init $\lambda_{\mathrm{o}}, \Delta \lambda, \delta_{\lambda}$

2. $x=\Delta \lambda+\delta_{\lambda}$

3. Estimate oscillation frequency

$$
\lambda_{\mathrm{t}}(\mathrm{t})=\lambda_{\mathrm{o}}+\mathrm{x}
$$

4. Add random error

$$
\lambda_{\mathrm{t}}(\mathrm{t})=\lambda_{\mathrm{t}}(\mathrm{t})+\varepsilon_{\lambda}(\mathrm{t})
$$

5. Evaluate test-observation time

$$
h_{i}(t)=h_{i}(0)+(1+y) \cdot t
$$

6. Perform normalization

$$
h_{i}(t)=O_{i}+S_{i} . t
$$

\section{End}

\section{2) Algorithm for Constructing Delay-based message}

The proposed system performs minimization of the synchronization error by interchanging the control message among the sensors with incorporated information of delay. Normally, the sensors uses control message for performing for perform either route discovery or for using it as acknowledgement for certain message delivery process. In the process of construction of control message, cluster head $(\mathrm{CH})$ plays a big contributory role. The algorithm can process any one form of input at a time i.e. $\mathrm{m}_{1}$ (message for selection of $\mathrm{CH}), \mathrm{m}_{2}$ (message for request to perform synchronization), and $\mathrm{m}_{3}$ (response message for synchronization) as shown in line-1. It is defining that control message should take up positive sign if the message of type $m_{1}$ is of query type or else it takes negative sign of the same message $m_{1}$ represents selection of cluster head (line-2). The algorithm than considers all the communicating nodes (line-3), where the node sends request message $m_{2}$ to the cluster head (line-4), which upon receiving forwards the response message $\mathrm{m}_{3}$ to node $i$. Finally, where the response is received by the node $i$, both node $i$ and cluster head interchanges more information about the local time parameter lt. The local time parameter is a set of local time that records timestamp at message forwarding process, message receiving process, response forwarding process, and time at which the cluster head forwards the response.

\section{Algorithm for Constructing Delay-based Control Message}

Input: $\mathrm{m}_{1}$ (message for selection of $\mathrm{CH}$ ), $\mathrm{m}_{2}$ (message for request to perform synchronization), $\mathrm{m}_{3}$ (response message for synchronization), lt (local time stamping parameters)

Output: constructing and disseminating control message

\section{Start}

1. init $\mathrm{m}_{1}, \mathrm{~m}_{2}, \mathrm{~m}_{3}, l t$

2. define $\mathrm{msg}[$ query $] \rightarrow-\mathrm{m}_{1} \| \mathrm{msg}[$ select $] \rightarrow+\mathrm{m}_{1}$

3. for $\mathrm{i}=1: \mathrm{n}$

4. $\quad \mathrm{i} \rightarrow \mathrm{m}_{2}\left(\operatorname{req}\left(\mathrm{d}_{\mathrm{i}}\right)\right) \rightarrow \mathrm{CH}$

5. $\quad \mathrm{CH} \rightarrow \mathrm{m}_{3}\left(\operatorname{res}\left(\mathrm{d}_{\mathrm{i}}\right) \rightarrow \mathrm{i}\right.$

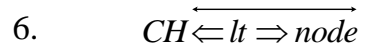

7. End

\section{End}

\section{3) Algorithm for Delay-based Routing}

The prior two algorithm can easily formulate a message and incorporate in enhancing time synchronization in sensor network considering delay-factor. However, this algorithm is responsible for constructing routes from node to cluster head that finally leads to base station. This algorithm will use a probability theory and network dynamics to estimate delay and then perform compensation of the computed delay while performing routing as well as minimization of synchronization errors. In order to advocate routing in sensor network, we understand that selection of cluster head has been always an essential part of data dissemination process of any wireless sensor network. However, the novelty of an approach is that consider cluster head to perform parallel task apart from data aggregation in the sensor network. In design, cluster head is also responsible for performing flooding operation for its timestamp values in the same simulation area. In proposed system, a node performs broadcasting of the beacons essentially to explore the identity-related information of the cluster head.

The first step of this algorithm basically works in this manner: A sensor broadcast the test beacons and computes the preliminary end-to-end delay after receiving the acknowledgement (or response). Till the computation of preliminary delay, the node doesn't send any other forms of message or data packets. After that the node concatenates the message with identity of the clusterhead. Its compute the delay factor by estimating time spend by both cluster head (to 
disseminate query message) and member node (to forward query message to its clusterhead itself) (Line-1-5). However, in case of maximum delay, there is a potential probability that the sensor queried for the clusterhead will not receive any response (Line-7). In such case of maximum delay, the sensor performs re-broadcasting of the beacons $\left(\mathrm{m}_{1}\right)$. Although the objective of this node is to develop a delay-aware route from a sensor to a cluster head but also will put a condition here for selection of energy-efficient clusterhead. Therefore, only the clusterhead whose residual energy $E_{\text {res }}$ is found to be more than certain cutoff level will be considered to perform routing with the enquired sensor. However, when the response is received from the clusterhead, the sensor checks for the identity of clusterhead.

\section{Algorithm for Delay Based Routing}

Input: $\mathrm{m}$ (message, $\mathrm{m}_{1}, \mathrm{~m}_{2}, \mathrm{~m}_{3}$ ), $\mathrm{CH}_{\mathrm{ID}}$ (clusterhead ID)

Output: Delay-based routes

\section{Start}

1. $\mathrm{i} \rightarrow \mathrm{m}_{1}\left(-1\right.$ concat $\left.\mathrm{CH}_{\mathrm{ID}}\right) / /$ broadcast

2. if $\left(\mathrm{i}\left(\mathrm{m}_{3}\right)=0\right)$

3. $\mathrm{i} \rightarrow \mathrm{m}_{1} / /$ re-broadcast

4. $\mathrm{i} \leftarrow \mathrm{m}_{3}\left(\mathrm{CH}\left(\mathrm{E}_{\mathrm{res}}>\mathrm{Th}\right)\right.$

5. save $\mathrm{CH}_{\mathrm{ID}}$

6. End

7. For $(\max (d))$

8. if $\left(\mathrm{CH}_{\mathrm{ID}} \sim-\mathrm{ve}\right)$

9. $\mathrm{m}_{1}=$ query $\mathrm{msg}$

10. else

11. $\mathrm{m}_{1}=$ selection of $\mathrm{CH}$

12. routeConstruct $\left(\mathrm{CH} \rightarrow\right.$ broadcast $\left.\left(\mathrm{CH}_{\mathrm{ID}}\right)\right)$

13. Remove dup(m)

14. End

End

However, if the sensor finds presence of any non-positive values in the inbound delay-aware message, it is considered to be a query message. In such case, if the $\mathrm{CH}_{\mathrm{ID}}$ is already with the sensor that it directly constructs a route between them. But in case the inbound delay-aware beacon consists of a positive values, than the beacon is considered to be announcement message, which will be again required to be transmitted in the network. In this case of the sensor doesn't have pre-defined information of $\mathrm{CH}_{\mathrm{ID}}$ (from prior routing table) than it just add it to its tree list and then performs broadcasting of message. The final step will be to construct routes and check for duplicated message in order to remove it. This task is carried out by receiver.

\section{4) Algorithm for Delay Compensation}

The proposed system considers a unavoidable network parameter called as delay in order to correctly perform time synchronization in large scale wireless sensor network. One of the interesting points to be noted in this regard is that it is perform computation of the amount of delay, than perform routing. While performing routing, the algorithm also considers compensating an adverse effect of drift of clock as well as offset of clock. This algorithm is a complementary algorithm in proposed routing mechanism. In this process, the sensor will need to scrutinize the inbound message type. In case of positive acknowledge of receiving message, the sensor mark the receiving time as $\mathrm{T}_{\text {rec }}$. However, if the other node is not a member node but a clusterhead than its forwards a response beacon that consist of local time stamping parameters lt. The part of the algorithm is the computation of two new variables of difference in time stamps i.e. $\alpha$ and $\beta$.

\section{Algorithm for Delay Compensation}

Input: $\mathrm{T}_{\mathrm{rec}}$ (receiving time), $\mathrm{T}_{\text {for }}$ (timestamp at message forwarding process), $\mathrm{T}_{\text {res }}$ (response forwarding process), Output: Delay compensation

\section{Start}

1. $\mathrm{i} \leftarrow \mathrm{m}_{3}$

2. Estm $\mathrm{T}_{\text {rec }}$

3. If responseGenerator $=\mathrm{CH}$

4. forward lt to $\mathrm{CH}$

5. End

6. estimate delay

$$
\Delta=\frac{\alpha-\beta}{2}
$$

7. Optimized delay,

$$
\mathrm{T}_{\mathrm{CH} \_ \text {res }}=\mathrm{T}_{\mathrm{CH} \_ \text {res }}+\Delta
$$

8. Delay compensation

$$
\mathrm{T}_{\text {delay_compen }}=\mathrm{T}_{\mathrm{CH} \_ \text {res }}+\left(\mathrm{T}_{\text {res }}-\mathrm{T}_{\mathrm{loc}}\right)
$$

9. Apply Algorithm for Delay-based routing

\section{End}

Basically, it compute the variable $\alpha$ as an absolute difference of timestamp at message receiving process $\left(\mathrm{T}_{\text {rec }}\right)$ and timestamp at message forwarding process $\left(\mathrm{T}_{\text {for }}\right)$. Whereas, the other variable $\beta$ can be computed as mean difference of local time and timestamp for response forwarding process $\left(\mathrm{T}_{\text {res }}\right)$. After the estimation of the network delay $\Delta$ due to time synchronization in line- 6 , the process attempts to add $\left(\mathrm{T}_{\mathrm{CH}_{-} \text {res }}\right)$ time at which the cluster head forwards the response (line-7). This adds further optimization part that can be used for minimizing the delay. Finally, perform exact amount of delay (Line-8), which has to be compensated in the entire process of routing continued by prior delay-based algorithm for routing (line-9).

\section{RESULT DISCUSSION}

This section discusses about the outcomes accomplished from the proposed study. The algorithms discussed in the prior section are implemented on Matlab on simple 32 bit windows 
environment. The simulation parameters considered for the proposed study is highlighted in Table 2.

TABLE II. ADOPTED SIMULATION PARAMETERS

\begin{tabular}{|l|l|}
\hline Parameter & Value \\
\hline Network area( Simulation) area & $1000 \times 1200 \mathrm{~m}^{2}$ \\
\hline Nodes & $50-500$ \\
\hline Clock speed & $3 \times 10^{3} \mathrm{~Hz}$ \\
\hline Simulation Iterations & 1000 \\
\hline Path loss exponent & 0.5 \\
\hline MAC Type & 802.11 \\
\hline Traffic Model & CBR \\
\hline Channel capacity & $300 \mathrm{kbps}$ \\
\hline Channel sensing time & $0.2 \mathrm{sec}$ \\
\hline Control packet size & $32 \mathrm{bits}$ \\
\hline Data packet size & $2000 \mathrm{bytes}$ \\
\hline Antenna Model & Omni-directional \\
\hline Transmission range & $10 \mathrm{~m}$ \\
\hline Transmission Energy consumption & $0.5 \mathrm{~J}$ \\
\hline Receiving Energy consumption & $0.25 \mathrm{~J}$ \\
\hline Ideal mode Energy consumption & $0.035 \mathrm{~J}$ \\
\hline Sleep mode Power consumption & $0.02 \mathrm{~J}$ \\
\hline Initial battery Energy of each node & $10 \mathrm{~J}$ \\
\hline
\end{tabular}

In order to perform comparative analysis, choose to compare proposed OTSA with prior technique of time synchronization viz. TFTS [25] and $\mathrm{E}^{2} \mathrm{TS}$ [29]. The rationale behind this selection for comparative analysis is discussed as below:

1) Reason for selecting TFTS [25]: We have modelled TFTS [25] for the reason that it is one of the techniques that provides three different types of algorithm in order to meet three different circumstances of time synchronization in sensor network. Designed using probabilistic technique, TFTS [25] uses graph theory to provide hypothetical time synchronicity, empirical time synchronicity, and optimized time synchronicity. A closer look into the outcome of TFTS [25] will show that it is scalable and have exhibited good performance in form of error minimization of time synchronicity. However, TFTS [25] couldn't answer how much energy efficient it is or how much capable it is to control the communication overhead. It is quite understood that maximum of the routing protocols in sensor network works on retransmission scheme with increase of rounds. This phenomenon will lead to formation of duplicated message during data aggregation which will definitely increase the communication overhead. Hence, it choose TFTS [25] to understand its effectiveness with rest of the protocols with new performance parameters.

2) Reason for selecting $E^{2} T S$ [29]: have designed E2TS [29] in order to overcome the energy gaps in the prior TFTS
[29]. Using standard radio-energy model, we develop a simple modelling of clock along with framing up the control message. The prime focus was laid on the minimizing i) time synchronization errors and ii) energy consumption in sensor network. The mathematical modelling involved in $\mathrm{E}^{2} \mathrm{TS}$ [29] uses clock drift, offset, and skew for designing the modelling of hardware clock. It also considers formulation of a control message with source node ID, message ID, and intermediate hop ID, destination node ID, and time factor. The outcome of the $\mathrm{E}^{2} \mathrm{TS}$ [29] was also compared with the standard techniques of time synchronization to find it more energy efficient with less errors. However, this model has more scope of improvement if the modelling is carried out by considering the delay factor. As stated in algorithm implementation section, consideration of transmission delay has a significant effect on both clock drift and skew. The analysis of the situation becomes more challenging if the sensor nodes works on multihop communication base leading impediment to accuracy rate. Hence, E2TS may claim better energy efficiency, but strongly felt that its capability to minimize synchronization error could be more enhanced. Therefore, it can be said that proposed study of OTSA is an enhanced work of $E^{2}$ TS [29].

3) Reason for selecting PulseSync [21]: This protocol was found to offer a better scalability specifically designed for distributed and large scale wireless sensor network. PulseSync is the latest protocol to overcome the accuracy problems in other flooding based approaches in time synchronization in sensor network. The technique uses sleep scheduling for energy conservation. An adverse effect of propagation time was found to be reduced by MAC layer based time stamping. The author also uses regression and flooding technique. However, it was found that skew value was kept very small initially in this work inspite of computing the original value. Unfortunately, PulseSync is not fault tolerant with its scalability property till questionable. Moreover, PulseSynce was only tested for minimized errors in time synchronization and it doesn't really prove its extent to communication overhead or computational complexity.

Therefore, it implement all the above stated techniques of time synchronization using common simulation parameters stated in Table 1 with respect to proposed OTSA which performs three different task of i) delay-based routing ii) enhancing network lifetime, and ii) optimizing time synchronous in large scale sensor network. Table 3 shows the numerical outcome accomplished after implementing the proposed OTSA along with other existing techniques for comparative performance analysis with respect to overhead, synchronous error, energy, and processing time. 
TABLE III. NUMERICAL OUTCOMES OF COMPARATIVE ANALYSIS

\begin{tabular}{|c|c|c|c|c|c|}
\hline & & \multicolumn{3}{|c|}{ Existing System } & \multirow[b]{2}{*}{ OTSA } \\
\hline \multirow{11}{*}{ 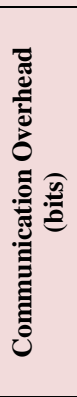 } & No. of Nodes & PulseSync [21] & TFTS [25] & $\mathbf{E}^{2} \mathrm{TS}$ [29] & \\
\hline & 50 & 20.21 & 14.26 & 12.35 & 12.34 \\
\hline & 100 & 25.38 & 17.28 & 14.28 & 14.26 \\
\hline & 150 & 27.41 & 21.76 & 19.76 & 18.77 \\
\hline & 200 & 30.01 & 28.76 & 25.26 & 18.97 \\
\hline & 250 & 31.56 & 30.98 & 26.78 & 19.36 \\
\hline & 300 & 34.76 & 32.87 & 27.86 & 21.01 \\
\hline & 350 & 50.38 & 42.09 & 29.31 & 22.53 \\
\hline & 400 & 52.38 & 43.21 & 29.84 & 22.54 \\
\hline & 450 & 53.98 & 43.35 & 29.98 & 22.56 \\
\hline & 500 & 60.01 & 43.31 & 30.07 & 22.57 \\
\hline & & \multicolumn{3}{|c|}{ Existing System } & \multirow[b]{2}{*}{ OTSA } \\
\hline \multirow{12}{*}{ 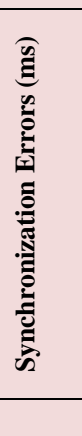 } & Iterations & PulseSync [21] & TFTS [25] & $\mathbf{E}^{2}$ TS [29] & \\
\hline & 100 & 0.752 & 0.535 & 0.419 & 0.534 \\
\hline & 200 & 0.751 & 0.527 & 0.417 & 0.534 \\
\hline & 300 & 0.746 & 0.519 & 0.408 & 0.512 \\
\hline & 400 & 0.703 & 0.479 & 0.374 & 0.415 \\
\hline & 500 & 0.693 & 0.489 & 0.369 & 0.409 \\
\hline & 600 & 0.638 & 0.497 & 0.355 & 0.351 \\
\hline & 700 & 0.617 & 0.462 & 0.345 & 0.317 \\
\hline & 800 & 0.599 & 0.428 & 0.329 & 0.302 \\
\hline & 900 & 0.555 & 0.362 & 0.318 & 0.203 \\
\hline & 1000 & 0.528 & 0.361 & 0.303 & 0.106 \\
\hline & & \multicolumn{3}{|c|}{ Existing System } & \multirow[b]{2}{*}{ OTSA } \\
\hline \multirow{12}{*}{ 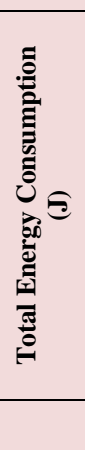 } & Data (bytes) & PulseSync [21] & TFTS [25] & $\mathbf{E}^{2} \mathrm{TS}$ [29] & \\
\hline & 1500 & 3.34 & 4.87 & 3.76 & 2.54 \\
\hline & 2000 & 3.45 & 4.89 & 3.87 & 3.32 \\
\hline & 2500 & 4.63 & 4.98 & 3.99 & 3.65 \\
\hline & 3000 & 4.79 & 5.28 & 4.25 & 3.74 \\
\hline & 3500 & 5.37 & 5.36 & 4.37 & 3.89 \\
\hline & 4000 & 5.39 & 5.55 & 4.55 & 3.98 \\
\hline & 4500 & 6.02 & 5.89 & 4.78 & 4.01 \\
\hline & 5000 & 6.77 & 6.32 & 5.29 & 4.1 \\
\hline & 5500 & 7.28 & 7.56 & 5.36 & 4.28 \\
\hline & 6500 & 8.99 & 7.59 & 5.59 & 4.29 \\
\hline & & \multicolumn{3}{|c|}{ Existing System } & \multirow[b]{2}{*}{ OTSA } \\
\hline \multirow{11}{*}{ 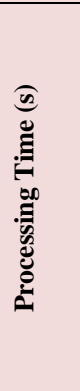 } & Data (bytes) & PulseSync [21] & TFTS [25] & $E^{2}$ TS [29] & \\
\hline & 1500 & 310 & 389 & 372 & 250 \\
\hline & 2000 & 363 & 392 & 375 & 251 \\
\hline & 2500 & 376 & 399 & 379 & 257 \\
\hline & 3000 & 389 & 417 & 389 & 269 \\
\hline & 3500 & 394 & 439 & 393 & 278 \\
\hline & 4000 & 410 & 463 & 410 & 287 \\
\hline & 4500 & 499 & 478 & 428 & 288 \\
\hline & 5000 & 519 & 482 & 455 & 292 \\
\hline & 5500 & 535 & 495 & 458 & 295 \\
\hline & 6500 & 555 & 501 & 471 & 299 \\
\hline
\end{tabular}

The above Table 2 shows the numerical outcomes for 4 different performance parameters e.g. communication overhead measured in bits, time synchronization errors measured in milliseconds, cumulative energy being consumed measured in Joule, and algorithm processing time measured in seconds. The numerical outcome obviously shows that proposed OTSA has outperformed the existing system. More discussion on the performance parameters are as follows:

\section{1) Analysis of Communication Overhead}

As the proposed system performs time synchronization based on delay-based routing, hence it bears a strong relationship with communication overhead. With more number of sensors adding, overhead could be anticipated to be more. The graphical outcome shown in Fig. 2 exhibits that PulseSync [21] posses significant communication overhead which is due to incapability of supporting network dynamics and multihop communications in sensor network. TFTS [25] is found lower communication overhead owing to its supportability of multihop network. However, TFTS [25] can likely show the hypothetical outcomes in case of dynamic traffic, which is overcome using $E^{2}$ TS [29]. $E^{2}$ TS [29] significantly considers authenticating the messages for time synchronous and thereby controls the overhead to a very large extent. OTSA further 
enhances this using optimization technique where transmission delay was emphasized in conjunction with routing approach supporting multihop network with network dynamics.

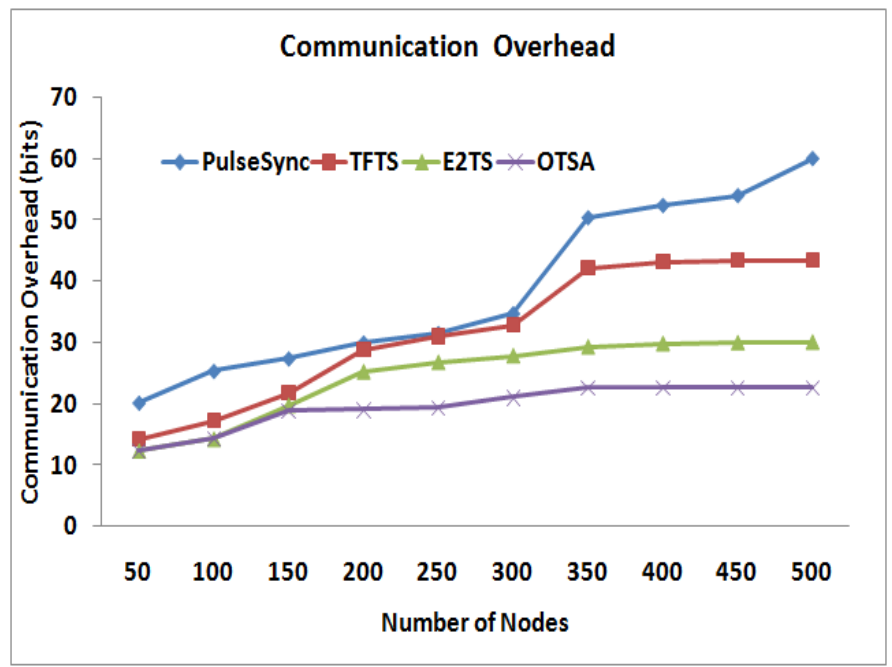

Fig. 2. Analysis of Communication Overhead

\section{2) Analysis of Synchronization Errors}

Fig.3 showcases the synchronization errors with increasing iterations (100-1000). The outcome clearly shows that OTSA has significant improvement with respect to existing protocols. PulseSync [21] has somewhat pattern of better descend in its curve, but owing to significant overhead its further scope for minimizing errors cannot be further optimized. TFTS [25] and $E^{2} T S$ [29] was found with better error minimization capabilities owing to its design flexibility, message formulations of exchanging the synchronous time. Similarly, proposed system of OTSA has further enhancement by incorporating multiple mechanism of evaluating local time stamping procedures. An error minimization process was completely based on the three different forms of control message for time synchronization during clustering and data aggregation stage that results in effective computation of actual and run-time delay causing the network to respond faster. Hence OTSA exhibits less error in time synchronization compared to existing approaches.

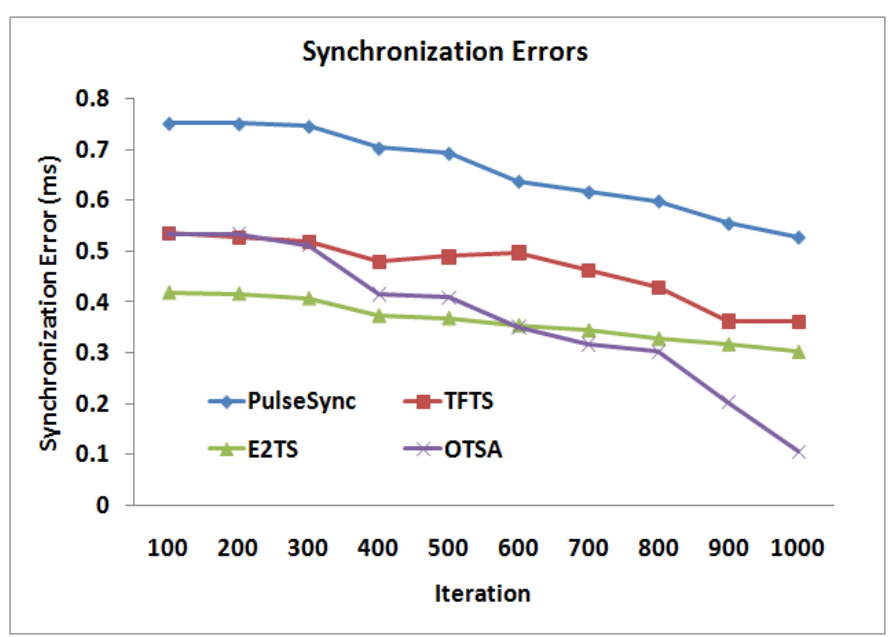

Fig. 3. Analysis of Synchronization Error

\section{3) Analysis of Network Lifetime}

Establishing a better balance between communication (with less time synchronous error) and energy consumption is one of the challenging tasks which is addressed in OTSA using delaybased routing approach. Using a simple first order radio model, OTSA only uses transmittance energy after the estimation of the synchronous error is computed which is carried out in the route discover process itself. Hence, transmittance time is almost unchanged and is not affected even in increasing number of the inbound data to be aggregated in OTSA. Hence, OTSA provides better network lifetime. However, PulseSync [21] performs extra processing almost in all the steps of data aggregation just to ensure drift minimization, which drains more energy. The prior TFTS [25] and $E^{2}$ TS [29] scheme also has better supportability of energy efficiency as compared to PulseSync [21]. However, OTSA has significant reduction $\mathrm{f}$ skew resulting is lesser probability of retransmission event, which significant maximum extent of energy consumption in OTSA. Therefore, it exhibits linearity in its controlling strategies for energy consumption.

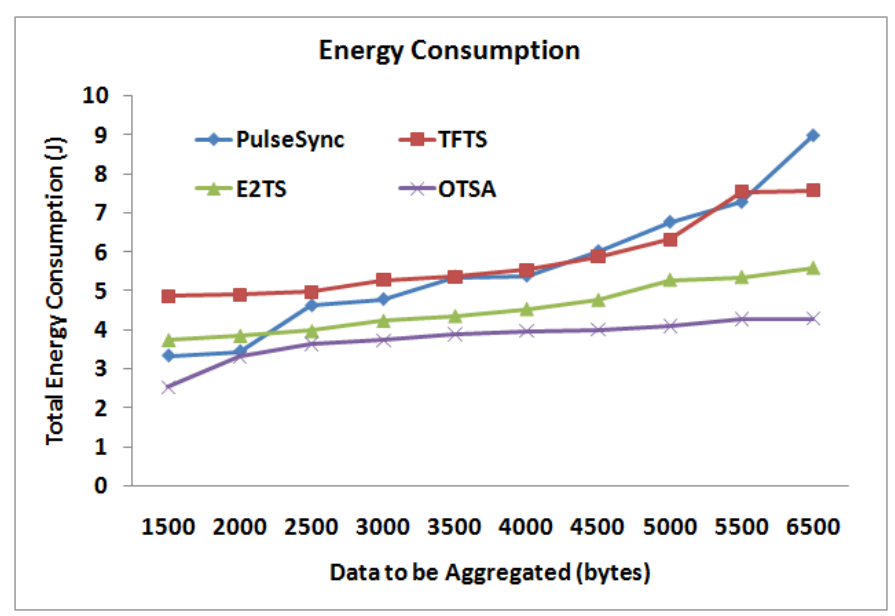

Fig. 4. Analysis of Energy Consumption

\section{4) Analysis of Processing Time}

Algorithm processing time plays an essential performance parameter to justify its effectiveness as sensors have lesser computational capability with availability of resources. It is quite natural that processing time will always have increasing trends with increasing number of the data packets to be processed during data aggregation. The processing time of PulseSync [21] was found to be almost linearly increasing which also states why PulseSync consumes more energy. Similarly, prior TFTS [25] and $\mathrm{E}^{2} \mathrm{TS}$ [29] has been witnessed with faster convergence time owing to usage of graph to be working on multihop networks. OTSA provides further improvement by considering delay factor, which is noniterative in nature causing the algorithm to converse faster. Hence, algorithm processing time is quite lower in proposed OTSA as compared to existing approaches. 


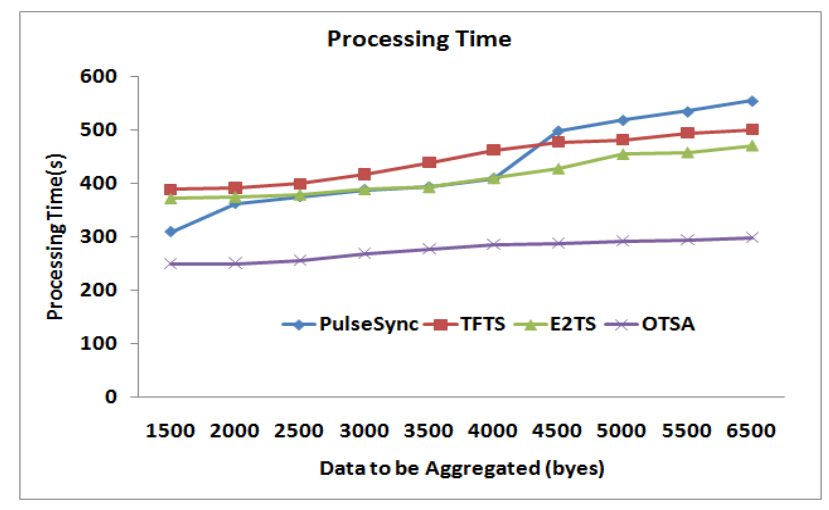

Fig. 5. Analysis of Processing Time

\section{CONCLUSION}

The proposed system has presented a novel technique those combinely addresses the problems of clustering, selection of clusterhead, routing, time synchronization. This study is the first attempt to address all the above stated problems in one technique. It use first order radio model and developed a technique which allows a node to live longer. It uses a threshold-based technique which ensures whenever a clusterhead reaches the cut-off values of threshold energy level; it converts itself to member node, which is quite different than existing techniques of clustering. Also, develop a technique that allows the sensor to share the time stamp information of the local clock in the broadcasting stage itself. Using this technique, the sensors (be it member node or clusterhead) are in better position to formulate a routing protocol by estimating delay. This design principle has a significant benefit that allows the sensor to rectify the errors in synchronization and then perform forwarding of the data. The best part of the technique is its capability to forward the data packet with extremely less probability of the packet drop that could lead to retransmission. The outcomes of the study is found to provide superior outcomes with respect to communication overhead, synchronization errors, extent of energy consumption, and algorithm processing time.

\section{REFERENCES}

[1] A. Koubaa, J.R. M. Dios, Cooperative Robots and Sensor Networks, Springer, 2015

[2] B. N. Klein, On the Importance of Time Synchronization for Context Aware Applications, kassel university press, 2011

[3] S. Rahamatkar, A Light Weight Time Synchronization Approach in Sensor Network, Lap Lambert Academic Publishing, 2012

[4] E. D. Knapp, J. T. Langill, Industrial Network Security: Securing Critical Infrastructure Networks for Smart Grid, SCADA, and Other Industrial Control Systems, Syngress, 2014

[5] M. Kezunovic, S. Meliopoulos, Vaithianathan Venkatasubramanian, Vijay Vittal, Application of Time-Synchronized Measurements in Power System Transmission Networks, Springer, 2014

[6] K. Nagarathna, J. D.Mallapur, "An Investigational Analysis of different Approaches and Techniques for Time Synchronization in Wireless Sensor Network", International Journal of Computer Applications, Vol.103, No.5, October 2014

[7] H. Xiong, Z. Chen, W. An, and B. Yang, "Robust TDOA Localization Algorithm for Asynchronous Wireless Sensor Networks", Hindawi Publishing Corporation, International Journal of Distributed Sensor Networks, 2015

[8] Y. Xing, Y. Chen, W. Yi, and C. Duan, "Time Synchronization for Wireless Sensor Networks Using Adaptive Linear Prediction", Hindawi
Publishing Corporation, International Journal of Distributed Sensor Networks, 2015

[9] W. Ting, C. C. Yang, G. Di, T. X. Ming, and W. Heng, "Clock Synchronization in Wireless Sensor Networks: A New Model and Analysis Approach Based on Networked Control Perspective", Hindawi Publishing Corporation Mathematical Problems in Engineering, 2014

[10] S. Kim and Y. Yoo, "SLSMP: Time Synchronization and Localization Using Seawater Movement Pattern in Underwater Wireless Networks", Hindawi Publishing Corporation International Journal of Distributed Sensor Networks, 2014

[11] M. Jin, D. Fang, X. Chen, Z. Yang, C. Liu, and X. Yin, "Voltage-Aware Time Synchronization for Wireless Sensor Networks", Hindawi Publishing Corporation, International Journal of Distributed Sensor Networks, 2014

[12] Z. Dengchang, A. Zhulin, and X. Yongjun, "Time Synchronization in Wireless Sensor Networks Using Max and Average Consensus Protocol", Hindawi Publishing Corporation, International Journal of Distributed Sensor Networks, 2013

[13] J. Wang, S. Zhang, D. Gao and Y. Wang, "Two-hop time synchronization protocol for sensor networks", Springer- EURASIP Journal onWireless Communications and Networking, vol.39, 2014.

[14] S. P. Ardakani, J. Padget, and M. D. Vos, "HRTS: a Hierarchical Reactive Time Synchronization protocol for wireless sensor Networks", Springer-lecture notes of Institute for Computer Sciences, Social Informatics, and telecommunication Engineering, pp.47-62, 2014

[15] M. S. Stankovi, S. S. Stankovi,_K. H. Johansson, "Distributed Time Synchronization in Lossy Wireless Sensor Networks", Distributed Time Synchronization in Lossy Wireless Sensor Networks, Estimation and Control of Networked Systems, Vol.3, Part.1, 2012

[16] S. Youn, "A Comparison of Clock Synchronization in Wireless Sensor Networks", Hindawi Publishing Corporation International Journal of Distributed Sensor Networks, 2013

[17] B. Kerkez, "Adaptive Time Synchronization and Frequency Channel Hopping for Wireless Sensor Networks", Technical Report of University of California at Berkeley, 2012

[18] F. Wang, P. Zeng, H. Yu, and X. Zhao, "A Reliable Time Synchronization Protocol for Wireless Sensor Networks", Proceedings of the Third International Symposium on Computer Science and Computational Technology, pp. 009-013, August 2010.

[19] W. Ikram, I.Stoianov, N. F. Thornhill, "Towards a Radio-Controlled Time Synchronized Wireless Sensor Network: A Work in-Progress Paper", IEEE Conference on Emerging Technologies and factory Automation, pp.1-4, 2010

[20] G. C. Gautam, T. P. Sharma, "A Comparative Study of Time Synchronization Protocols in Wireless Sensor Networks, International Journal Of Applied Engineering Research, Dindigul, Vol.1, No 4, 2011

[21] C. Lenzen, P. Sommer, and R. Wattenhofer, "PulseSync: An Efficient and Scalable Clock Synchronization Protocol," IEEE/ACM Transaction on Networking, pp.717-727, 2014

[22] D. S. Sanchez, L. Alonso, P. Angelidis, and C. Verikoukis, "Secure Precise Clock Synchronization for Interconnected Body Area Networks", Hindawi Publishing Corporation EURASIP Journal on Wireless Communications and Networking, 2011

[23] L. Tang, Y. Sun, O. Gurewitz, D. B. Johnson, "EM-MAC: A Dynamic Multichannel Energy-Efficient MAC Protocol for Wireless Sensor Networks" Proceedings of the Twelfth ACM International Symposium on Mobile Ad Hoc Networking and Computing, No.23, 2011

[24] R. Albu, Y. Labit, T. Gayraud, P. Berthou, "An Energy-efficient Clock Synchronization Protocol for Wireless Sensor Networks", IEEE Wireless days, pp.1-5, 2010

[25] K.Nagarathna and J.D.Mallapur "TFTS:A Novel Triple Factor Time Synchronization for Effective Routing in Large Scale WSN" An International Journal of Wireless Personal Communication, Vol.84, No2, 2015

[26] KNagarathna and J.D.Mallapur "An Optimized \& On-Demand Time Synchronization in Large Scale Wireless Sensor Network: OOD-TS" Springer India, Emerging Research in Computing Information and Communication and Applications, DOI 10.1007/987-81-322-2550-8-29, 2015 
[27] J. D. Mallapur, M. I. Sataraddi, S. C. Hiremath and K. Nagarathna, "Priority Based Scheduler for Bluetooth Network", Proceedings of International Conference on Advances in Communication, Computing and Control, (ICAC3), Fr. Conceicao Rodrigues College of Engineering, Bandra, Mumbai, CCIS 361, pp. 356-365, 2013

[28] J D. Mallapur, Kiran Y. B, K. Nagarathna S. Hiremath "Trust Based Secured Routing in Wireless Multimedia Sensor Networks" Fourth International Conference on Computational Intelligence,
Communication Systems and Networks, 24 - 26 July Pucket , pp 53-58, 2012.

[29] K. Nagarathna and J. D. Mallapur " $E^{2} T S$ : Energy Efficient Time Synchronization Technique in Large Scale Wireless Sensor Network", Springer International Publishing Switzerland, Artificial Intelligence Perspectives and Applications, 225 Advances in Intelligent Systems and Computing 347, DOI: 10.1007/978-3-319-18476-0_23, 2015 